International Journal of Engineering \& Technology, $7(4.10)(2018) 440-442$
International Journal of Engineering \& Technology
SPC
Website: www.sciencepubco.com/index.php/IJET
Research paper

\title{
A Study on Slope Number of Certain Classes of Bipartite Graphs
}

\author{
A. Antony mary ${ }^{1 *}$, A. Amutha , M.S. Franklin Thamil Selvi \\ ${ }^{I}$ Assistant Professor, Department of Mathematics, Sathyabama Institute of Science and Technology, Chennai, India. \\ ${ }^{2}$ Assistant Professor, Department of Mathematics, The American College, Madurai, India \\ ${ }^{31}$ Assistant Professor, Department of Mathematics, Sathyabama Institute of Science and Technology, Chennai, India. \\ *Corresponding author E-mail: amuthajo@gmail.com,anto.doss@yahoo.com
}

\begin{abstract}
Graph drawing is the most important area of mathematics and computer science which combines methods from geometric graph theory and information visualization. Generally, graphs are represented to explore some intellectual ideas. Graph drawing is the familiar concept of graph theory. It has many quality measures and one among them is the slope number. Slope number problem is an optimization problem and is NP-hard to determine the slope number of any arbitrary graph. In the present paper, the investigation on slope number of bipartite graph is studied elaborately. Since the bipartite graphs creates one of the most intensively investigated classes of graphs, we consider few classes of graphs and discussed structural behavior of such graphs.
\end{abstract}

Keywords: crown graph; grid graph; hypercube; ladder graph; slope number; star graph; straight line drawing.

\section{Introduction}

Graphs are objects that models social networks, communication networks, social networks etc., and arises in other area of mathematics such as probability, geometry, number theory etc. The most common representation of a graph is by means of diagrams. Drawings of graphs have created extraordinary interest due to its extensive applications to explore different concepts and objects in computer architectures, VLSI circuits, networks etc. There are some aesthetic criteria to have some nice drawing such as straight line segments, few edge crossings, symmetry etc. In a straight line drawing, edges are drawn by straight line segments not necessarily by horizontal or vertical line segments.

The study of straight line drawing using few slopes is related to the study of the slope number. The slope of an edge in a straight line drawing is the family of all straight lines parallel to this edge [2]. Motivated by this fact, the slope number problem has been chosen for investigation. Wade and Chu were the first to define the slope number in 1994 [4]. Though it is NP-complete, it is NPhard problem to find the slope number of an arbitrary graph [1]. Bipartite graphs are extensively used in modern coding theory apart from being used in modeling relationship. In line of thought, we have chosen bipartite graphs for investigation. In the present paper, we have discussed the structural behavior of bipartite graphs and investigated the slope number of few bipartite graphs such as crown graph, hypercube, ladder graph, grid graph and star graph. This represents the slope number of bipartite graph with large and small slopes. Also the characterization of such graphs with respect to edges, diameter, edge coloring is explored.

\section{Overview of the Paper:}

A graph is an ordered pair $\mathrm{G}=(\mathrm{V}(\mathrm{G}), \mathrm{E}(\mathrm{G}))$ where $\mathrm{V}(\mathrm{G})$ is the vertex set whose elements are vertices and $E(G)$ is the edge set whose elements are edges. A graph of $\mathrm{H}=(\mathrm{V}(\mathrm{H}), \mathrm{E}(\mathrm{H}))$ is a subgraph of $\mathrm{G}$, if $\mathrm{V}(\mathrm{H}) \subseteq \mathrm{V}(\mathrm{G})$ and $\mathrm{E}(\mathrm{H}) \subseteq \mathrm{E}(\mathrm{G})$. In a graph $\mathrm{G}$, the greatest distance between any pair of vertices of $G$ is called the diameter of $\mathrm{G}$ and is denoted by $\operatorname{diam}(\mathrm{G})$. An edge coloring of $\mathrm{G}$ is a coloring of edges such that no two edges receive same color. A matching $M$ in $G$ is a sub-graph of $G$ such that every edge shares no vertex with any other edge. A maximum matching is the maximum cardinality of edges in M. In this paper, we presented the slope number which is large for hypercube and crown graph, since the structural behavior of the graphs are similar. Also, the slope number is discussed which is small for ladder graph, grid graph and star graph.

\section{Slope Number}

The slope number of a graph $\mathrm{G}$ is the minimum number of distinct edge slopes required to draw the graph $\mathrm{G}$

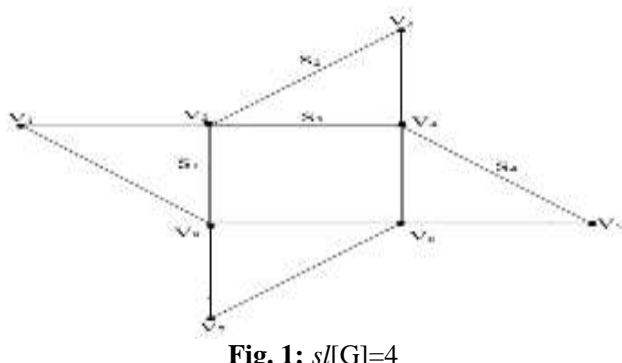


From the figure (1), Let $S_{1}$ be the slope of an edge $V_{2} V_{8}$. Let $S_{2}$ be the slope of an edge $V_{2} V_{3}$. Let $S_{3}$ be the slope of an edge $V_{2} V_{4}$ and $S_{4}$ be the slope of an edge $V_{4} V_{5}$. The slope number of the graph is 4 .

\subsection{Theorem [4]:}

Let $\mathrm{G}$ be a complete graph $\mathrm{K}_{\mathrm{n}}$ on $\mathrm{n}$ vertices. Then $s l[\mathrm{G}]=\mathrm{n}$, if $\mathrm{n} \geq 3$.

\section{Slope Number of Crown Graph:}

Crown graphs are of major attention in the concept of word representation graphs. Crown graph is a graph attained from the complete bipartite graph $\mathrm{K}_{\mathrm{n}, \mathrm{n}}$ by removing a horizontal edges and is denoted by $\mathrm{H}_{n, \mathrm{n}}$. The number of vertices in the crown graph $(\mathrm{n}>2)$ is $2 n$ and the number of edges is $n^{2}-n$. The maximum and minimum degree of the crown graph is $\mathrm{n}-1$. Therefore, every crown graph is a n-1 regular graph. The diameter of crown graph is 3 , if $n \geq 3$. Figure 2 is the crown graph on 8 vertices.

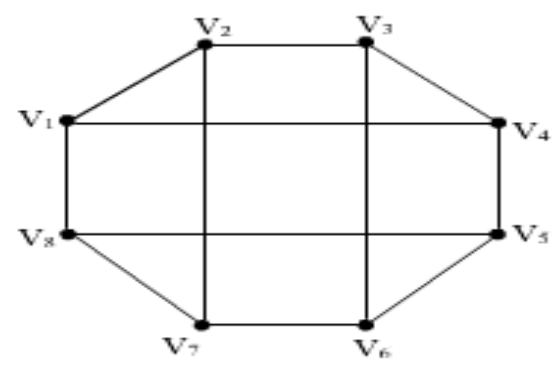

Fig. 2: Crown graph on 8 vertices

\subsection{Theorem:}

Every crown graph has exactly $\mathrm{n}$ distinct slopes i.e, $s l\left[\mathrm{H}_{\mathrm{n}, \mathrm{n}}\right]=\mathrm{n}$.

Proof:

Let $\mathrm{G}$ be crown graph on $2 \mathrm{n}$ vertices.

Arrange the vertices in a cyclic order. Label the vertices of $G$ as $\mathrm{V}_{1}, \mathrm{~V}_{2} \ldots \mathrm{V}_{2 \mathrm{n}}$ in a clockwise direction. Choose $\mathrm{V}_{\mathrm{i}}$ such that $1 \leq \mathrm{i} \leq \mathrm{n}$, which is the rightmost vertex, From $V_{i}$ draw the edges successively of length $2 \mathrm{n}$ which completes the cycle. Since each vertex of the cycle has degree 2 , it shares atmost $\mathrm{n}$ distinct slopes. It is known that $\mathrm{H}_{\mathrm{n}, \mathrm{n}}$ is $\mathrm{n}-1$ regular. Draw the edges parallel to the slopes of $G$. In order to preserve the degree of $H_{n, n}$ each pair of vertices uses the slopes which is already used by the vertex of the cycle. Hence $\mathrm{n}$ slopes suffice to draw G. $\therefore s l\left[\mathrm{H}_{\mathrm{n}, \mathrm{n}}\right]=\mathrm{n}$ Hence the proof.

\subsection{Theorem:}

Let $\mathrm{G}$ be a crown graph. Then slope of the graph is odd or even, if and only if it has even number of edges.

\section{Proof:}

Assume that $\mathrm{G}$ has $\mathrm{n}$ slopes (odd or even).

To prove that $\mathrm{G}$ has even number of edges.

Let $S=\left\{S_{1}, S_{2}, \ldots, S_{n}\right\}$ be $n$ slopes of the graph.

By theorem 4.1, $\operatorname{sl}\left[\mathrm{H}_{n, n}\right]=n$. Since the edges of the graph is $n^{2}-n$, the number of edges of $\mathrm{G}$ is always even.

\section{Conversely, Assume that number of edges of $\mathrm{G}$ is even.}

To prove that $\mathrm{G}$ has $\mathrm{n}$ slopes. Since the maximum degree of $\mathrm{H}_{\mathrm{n}, \mathrm{n}}$ is $\mathrm{n}-1$ regular, atmost two edges incident to the same vertex is distinct. It is known that $\mathrm{G}$ has even number of vertices. Therefore, the edges incident to each vertex of $\mathrm{G}$ has $\mathrm{n}$ slopes.

By theorem 4.1, $s l\left[\mathrm{H}_{\mathrm{n}, \mathrm{n}}\right]=\mathrm{n}$.

Hence the proof.

\subsection{Theorem:}

If $\mathrm{G}$ is a crown graph with $\mathrm{n}$ slopes, then $\mathrm{G}$ is $\mathrm{n}-1$ edge colorable.

Proof:

Assume that $G$ has $n$ slopes. Let $S=\left\{S_{1}, S_{2}, \ldots, S_{n}\right\}$ be $n$ slopes of the graph.

To prove that $\mathrm{G}$ is $\mathrm{n}-1$ colorable.

Since $\mathrm{G}$ is $\mathrm{n}-1$ regular, it admits $\mathrm{n}-1$ colours

$\Rightarrow \mathrm{G}$ is $\mathrm{n}-1$ colourable

Hence the proof.

\subsection{Observation:}

If $G$ is a crown graph with $\operatorname{diam}[G]=3$, then the slope number is always $n$.

\subsection{Theorem:}

Let $\mathrm{G}$ be a crown graph. Then $\alpha^{\prime}[\mathrm{G}]=\operatorname{sl}[\mathrm{G}]$.

\section{Slope Number of Hypercube:}

The $\mathrm{n}$-dimensional hypercube is a graph whose vertices are $2^{\mathrm{n}}$ and edges are $\mathrm{n} 2^{\mathrm{n}-1}$. It is a simple graph which consists of two copies of $\mathrm{Q}_{\mathrm{n}-1}$ connected together at corresponding nodes. The diameter of $\mathrm{Q}_{\mathrm{n}}$ is $\mathrm{n}$. Figure 3 is the hypercube on 8 vertices.

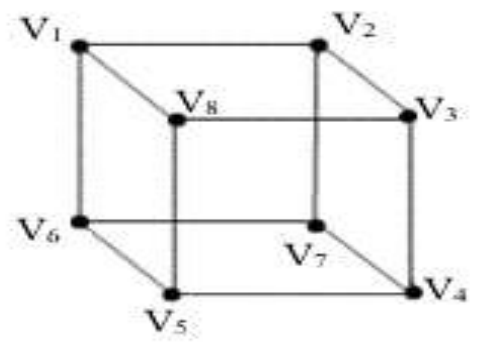

Fig. 3: Hypercube on 8 vertices

\subsection{Theorem [3]:}

If $\mathrm{Q}_{\mathrm{n}}$ is a graph of hypercube of dimension $\mathrm{n}$, then $\operatorname{sl}\left[\mathrm{Q}_{\mathrm{n}}\right]=\mathrm{n}$.

\subsection{Observation:}

Every n-dimensional hypercube of maximum degree has $\Delta(\mathrm{G})$ distinct slopes.

\subsection{Theorem:}

Let $\mathrm{G}$ be $\mathrm{n}$-dimensional hypercube. Then $s l[\mathrm{G}]=\mathrm{n}$, if and only if it has even number of edges.

\subsection{Theorem:}

If $\mathrm{G}$ is a hypercube of $\mathrm{n}$-dimension with $\mathrm{n}$ slopes, then $\mathrm{G}$ is $\mathrm{n}$-edge colorable.

\subsection{Observation:}

Let $\mathrm{G}$ be a hypercube of $\mathrm{n}$-dimension. Then $\operatorname{diam}[\mathrm{G}]=\Delta[\mathrm{G}]=\operatorname{sl}[\mathrm{G}]$

\subsection{Observation:}

Let $\mathrm{G}$ be $\mathrm{n}$-dimensional hypercube graph. Then $\alpha^{\prime}[\mathrm{G}]=2^{s l[\mathrm{G})-1}$. 


\section{Slope Number of Ladder Graph and Grid Graph.}

Grid graph is the cartesian product of path graphs on $m$ and $n$ vertices such that $P_{m} \times P_{n}$. It is denoted by $G_{m, n . .}$ The ladder graph is a special case of grid graph. The ladder graph is a graph which is a cartesian product of two path graphs such that $\mathrm{P}_{\mathrm{n}} \times \mathrm{P}_{2}$ and is denoted by $L_{n}$. The vertices and edges of $L_{n}$ are $2 n$ and $n+2$ edges. In a drawing of grid graphs and ladder graphs, only two slopes suffices i.e set of vertices passing through horizontal and vertical line segments. Refer figure 4 and figure 5.

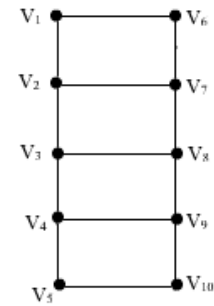

Fig. 4: Ladder graph on 10 vertices

\subsection{Theorem}

For every $\mathrm{n} \in \mathrm{N}, \operatorname{sl}\left[\mathrm{L}_{\mathrm{n}}\right]=\operatorname{sl}\left[\mathrm{G}_{\mathrm{m}, \mathrm{n}}\right]$

\subsection{Theorem:}

Let $\mathrm{G}$ be a ladder graph and grid graph. Then $s l[\mathrm{G}]=2$, if and only if it has odd or even number of edges.

\subsection{Theorem:}

If $L_{n}$ is a 2 slope graph, then $G$ is 3-edge colorable.

\subsection{Theorem:}

If $\mathrm{G}$ is a ladder graph with $\operatorname{diam}[\mathrm{G}]=\mathrm{n}$, then the slope number is always 2 .

\subsection{Observation:}

Let $\mathrm{G}$ be a ladder graph on $\mathrm{n}$ vertices. Then $\alpha^{\prime}[\mathrm{G}]=\operatorname{diam}[\mathrm{G}]$.

\section{Slope Number of Star Graph.}

A star graph is a graph on $n$ vertices, where $n$ vertex has degree $n$ 1 and the other vertices have degree 1 . It is denoted by $S_{n}$. The diameter of star graph is 2. Figure 6 is the illustration of star graph on 8 vertices.

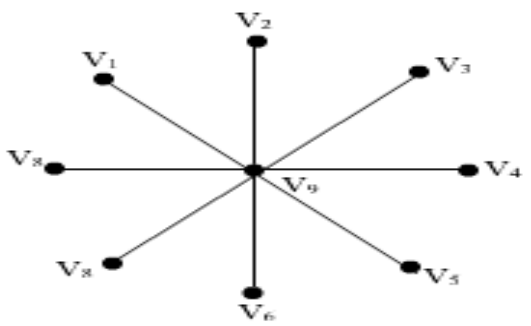

Fig. 6: star graph on 9 vertices

\subsection{Theorem:}

Let $\mathrm{G}$ be a star graph. Then $s l[\mathrm{G}]=\left\lceil\frac{n}{2}\right\rceil$, if and only if it has odd or even number of edges.

\subsection{Observation:}

Every star graph has $\left\lceil\frac{\Delta}{2}\right\rceil$ distinct slopes i.e $\operatorname{sl}\left[\mathrm{K}_{1, \mathrm{n}}\right]=\left\lceil\frac{\Delta}{2}\right\rceil$

\subsection{Theorem:}

Every star graph with $\left[\frac{n}{2}\right]$ slopes is n-edge colorable.

\subsection{Observation:}

Let $\mathrm{G}=\mathrm{K}_{1, \mathrm{n}}$, then $\operatorname{sl}[\mathrm{G}]=\left[\frac{n}{\operatorname{diam}(G)}\right]$.

\section{Conclusion:}

Thus we have showed the results on slope number of bipartite graphs with large and small slopes and its characterization on edges, diameter, edge coloring is also discussed. Still more properties and characteristics on slope number of other graphs are yet to be investigated. Also, this problem is worth considering for Cayley and Non-Cayley graphs.

\section{References:}

[1] Formann M, Hagerup T, Haralambides J, Kaufmann M, Leighton FT, Symvonis A, Welzl E \& Woeginger G, Drawing graphs in the plane with high resolution, SIAM Journal on Computing, 22 (5), (1993), pp. 1035-1052.

[2] Knauer K, Micek P \& Walczak B, Outer planar graph drawings with few slopes, Computational Geometry: Theory and Applications, 47, (2014), pp. 614-624.

[3] Pallavi Jain, Gursaran \& Kamal Srivastava, Slope number of some classes of graphs, International conference on mathematical sciences, (2014).

[4] Wade GA \& Chu JH, Drawability of complete graphs using a minimal slope set, The computer Journal, 37 (2), (1994), pp. 139-142.

\subsection{Theorem:}

Let $\mathrm{G}=\mathrm{K}_{1, \mathrm{n}}$. Then $s l[\mathrm{G}]=\left\lceil\frac{n}{2}\right\rceil$ 